\title{
The Role of Chinese Medicine in the Treatment of Chronic Diseases in China
}

Authors

Affiliations
Miao Jiang ${ }^{1}$, Chi Zhang ${ }^{1}$, Hongxin Cao ${ }^{1}$, Kelvin Chan ${ }^{2}$, Aiping Lu ${ }^{1}$

${ }^{1}$ Institute of Basic Research in Clinical Medicine, China Academy of Chinese Medical Sciences, Beijing, China

2 The University of Sydney and University of Western Sydney, Sydney, Australia
Key words

- chronic disease

- efficacy

- safety

- Chinese medicine

- China

\section{Abstract \\ $\nabla$}

Chinese medicine (CM) has a long history of experience and proven successful treatment for chronic diseases and has also played an important role in the provision of health care in China. Patients with chronic diseases are happy to accept $\mathrm{CM}$ and physicians are willing to use $\mathrm{CM}$ to relieve patients suffering from chronic illnesses. The Chinese health authorities encourage CM development to meet the requirements for the treatment of chronic diseases. CM products are an essential part of medications that have a predominant role in the prevention and treatment of chronic diseases in China. A large number of CM clinical studies, including a substantial number of available randomized controlled trials and systematic reviews, have shown that $\mathrm{CM}$ is effective and safe in the treatment of chronic diseases. Although the efficacies of some evaluated $\mathrm{CM}$ therapies remain

\section{Introduction}

\section{$\nabla$}

The long-term clinical practice of traditional Chinese medicine $(\mathrm{CM})$ confirms its importance and essential role in the health care system in China, especially in the prevention and treatment of chronic diseases [1]. With its unique theoretical system and accumulated clinical experiences, $\mathrm{CM}$ has developed a comprehensive therapeutic approach and gained the confidence of the public. More and more patients with chronic diseases prefer the CM treatment or the integration of $\mathrm{CM}$ treatment with biomedicine therapy. Although $\mathrm{CM}$ is currently facing some significant challenges from both the application of modern medicine and social development, its practice still accounts for around $20 \%$ of all health care delivered in China [2].

The nutritional status and sanitary conditions have been significantly improved for the people uncertain, it is worth assessing them by using $\mathrm{CM}$ pattern (Zheng or syndrome) differentiation to verify treatment outcomes. CM is considered to have a better safety profile compared to pharmaceutical chemicals, but inappropriate applications of CM also makes the safety issues a hot discussed subject. As a medical system, CM should be able to provide worldwide contribution for the patients who are suffering from chronic diseases. The application of CM pattern classification in diagnosis with corresponding prescribed treatment using herbal formulae in the relief of chronic diseases can be linked with modern biomedical parameters (biomarkers) as treatment outcomes. These outcome parameters, together with the patients' reported quality of life assessment, can provide innovative approaches for evidencebased estimation of the efficacy of CM treatment in chronic diseases.

in China, and the major causes of mortality have changed from infectious diseases to coronary heart disease and other chronic illnesses [3]. Meanwhile, the Chinese government has realized that an affordable and easily accessible health care system will be essential for the stability of the country. "The high cost to receive health care and the inconvenience to access it" have become two major complaints among Chinese people [4]. In an effort to address these concerns and provide high-quality, affordable, and accessible health care to Chinese people, the Chinese government has explored a number of approaches to remodel the health care system, which has been significantly reformed since the beginning of the 1980s [5], and new initiatives were launched in 2006, which are aimed to provide at least one community health care center for every 30000 to 100000 citizens. The centers will integrate Western medicine (WM, biomedicine) and CM, which 
will move CM into full application in the treatment of chronic diseases in China.

This paper is to review the role of $\mathrm{CM}$ in the treatment of chronic diseases in China and discuss the major issues in spreading the application of CM worldwide for this purpose.

\section{CM is an Important Health Care Approach in China $\nabla$}

There is a saying in China: CM is good at treating chronic diseases since it is focusing on the Ben (root) of the disease, and Western medicine (WM) is good at treating acute diseases since it is focusing on the Biao (phenomenon) of the disease. The distribution of patient visits in different departments in China hospitals in 2009 showed that over $30 \%$ of the patient visits (both of inpatients and outpatients) were found in the internal medicine departments (the majority of the patients had chronic diseases) in CM hospitals ( Fig. 1) [6], while patient visits to internal medicine departments of WM hospitals are remarkably lower (about 21-25\%). The data also showed that there were more than $10 \%$ patient visits to CM departments of general (WM) hospitals (12.7\% and $18.8 \%$ inpatients and outpatients, respectively). The results indicated that the patients with chronic diseases in China are willing to see CM doctors either in CM hospitals or in CM departments of WM hospitals.

Two surveys on the use of CM from patients with chronic diseases conducted by our team in Beijing from 2008 to 2009 also support that CM has been favored by these patients (unpublished data). The first survey was conducted on 968 patients with any of the 4 most common chronic diseases in China: diabetes, hypertension, coronary heart disease, and osteoporosis. The results show that more than half of the respondents believed that CM therapies could help them improve their health situation. The other survey was conducted on the health care professionals in community clinics in Beijing and focused on the major perception of the role of CM. A total of 306 medical staff members were included in the survey, and the majority expressed a desire to provide both WM and CM for the patients. All of them expressed fully confidence on the important role of $\mathrm{CM}$ in the prevention and treatment of chronic diseases. Furthermore, a total of 278 responders expressed their willingness to accept further CM training to improve their health care career. The results suggest that the role of $\mathrm{CM}$ in the treatment of chronic diseases retains a good image not only for the public in China but also among the health care professionals.

The data of recent years from the Ministry of Public Health of China shows that the quality and efficiency of the medical service in $\mathrm{CM}$ and WM hospitals are similar ( Fig. 2) [6,7]. However the average expenditure for both outpatient and inpatient in CM hospitals was lower than in WM hospitals ( Fig. 3), even though the average stay in hospital of inpatients in CM hospitals was longer than in WM hospitals ( Fig. 4). The data indicated that CM therapy is relatively cheaper than WM therapy. That might be one of the reasons why $\mathrm{CM}$ is favored both by patients with chronic diseases and professionals in community clinics and the Chinese authorities encourage the use of $\mathrm{CM}$ for the treatment of chronic diseases.

In the health care system reform of China, to set up the National Essential Drug List and Drug List for Insurance Coverage are primary steps towards the provision of accessible, affordable, effective, and safer health care for all. In the National Essential Drug List in China (issued in 2009 for community clinics), there are $102 \mathrm{CM}$ products out of total $307 \mathrm{drugs}$, and CM products are recommended to treat patients with chronic diseases. In the National Health Insurance Drug List (Version 2009), there are a total of $683 \mathrm{CM}$ products (also called proprietary Chinese medicines, PCM) which are mainly for internal medicine application. Among them, 103 are commonly used for the treatment of cardiovascular and cerebrovascular diseases. These essential policies on $\mathrm{CM}$ in China, which are based on its clinical effectiveness in a long
Percentage of Outpatients \& Inpatients in General Hospitals By Department
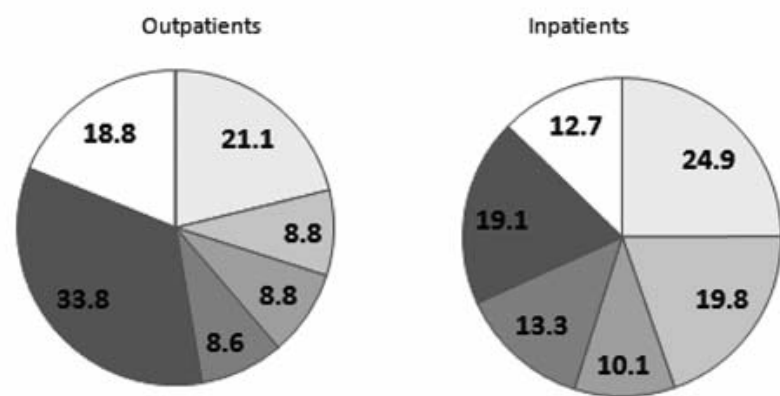

Percentage of Outpatients \& Inpatients in CM Hospitals By Department

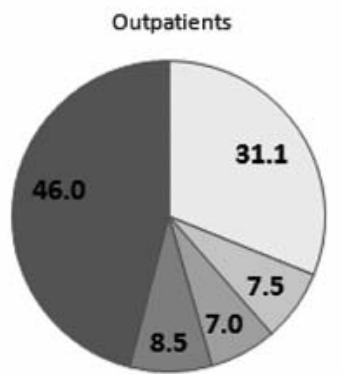

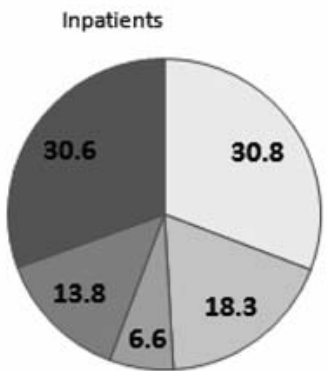

Fig. 1 Distribution of patient visits in different departments in WM and CM hospitals in the year of 2009. 

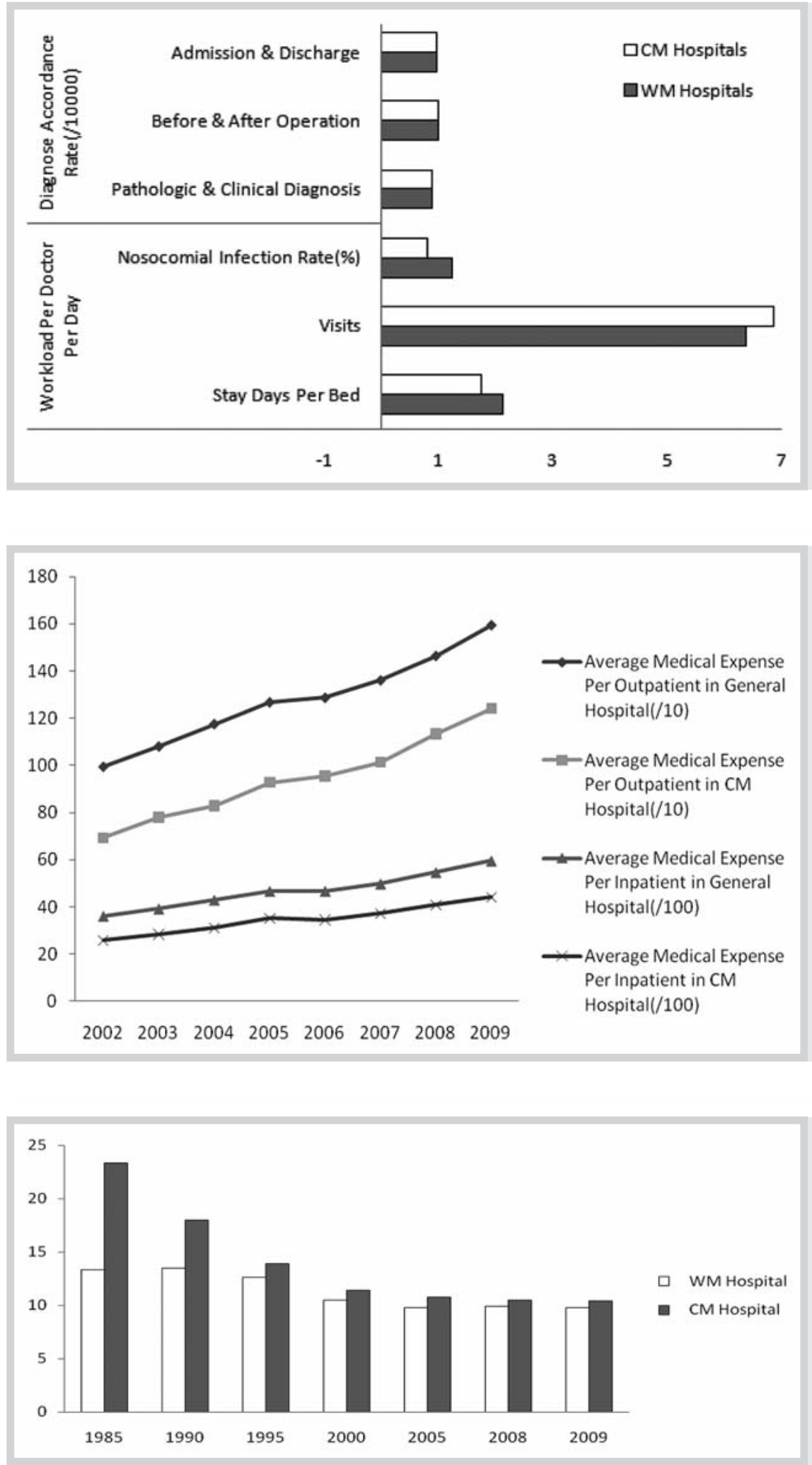

Fig.2 Quality and efficiency of the medical service in CM and WM hospitals in 2009.

Fig. 3 Average medical expense (Chinese RMB) per outpatient and inpatient in CM and WM hospitals.
Fig. 4 Average stay (days) in CM and WM hospitals. history, supply a solid base for further development and application of CM in the Chinese health care system.

Since China has good policies for CM development, there are steadily increasing trends in application, numbers of personnel, and medical services in China ( Figs. 5 and 6). China has been encouraging the development of $\mathrm{CM}$ products in the past 20 years. The data shows that the output value of the pharmaceuti- cal industry in CM in 2007 was up to 669.7 billion RMB (Yuan) which amounts to $26.4 \%$ of the total value for the pharmaceutical industry in China. On the other hand, there are much more functional foods developed from CM in China. A study shows that, as of the end of September 2010, China had totally reviewed 10118 functional food applications, and most of them are formulated with Chinese Materia Medica (CMM) ingredients. Among them, 


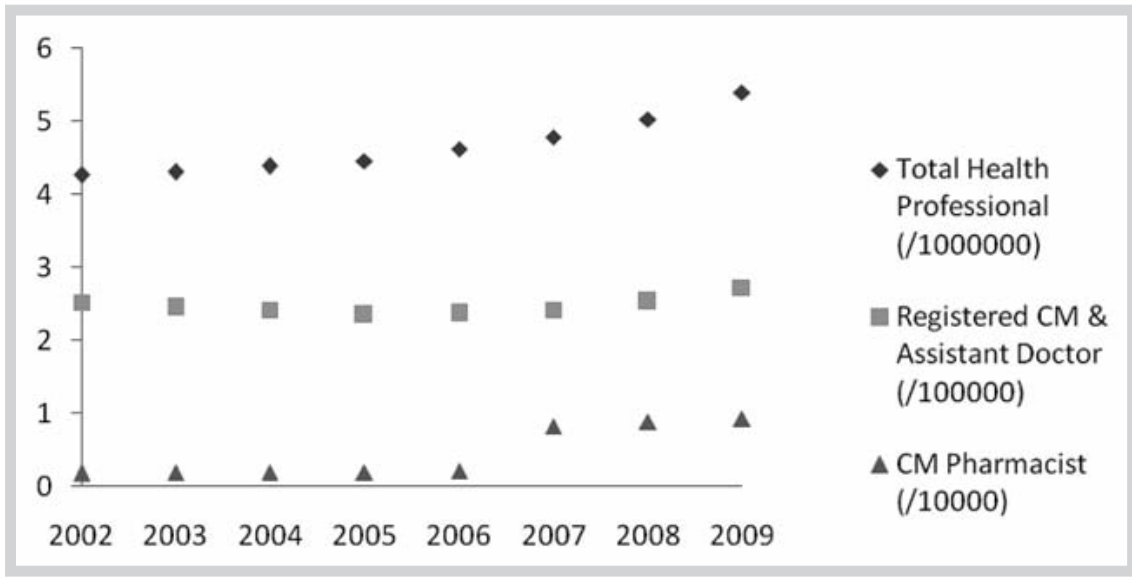

Fig. 5 The ratios of CM personnel to total health care professionals in China.

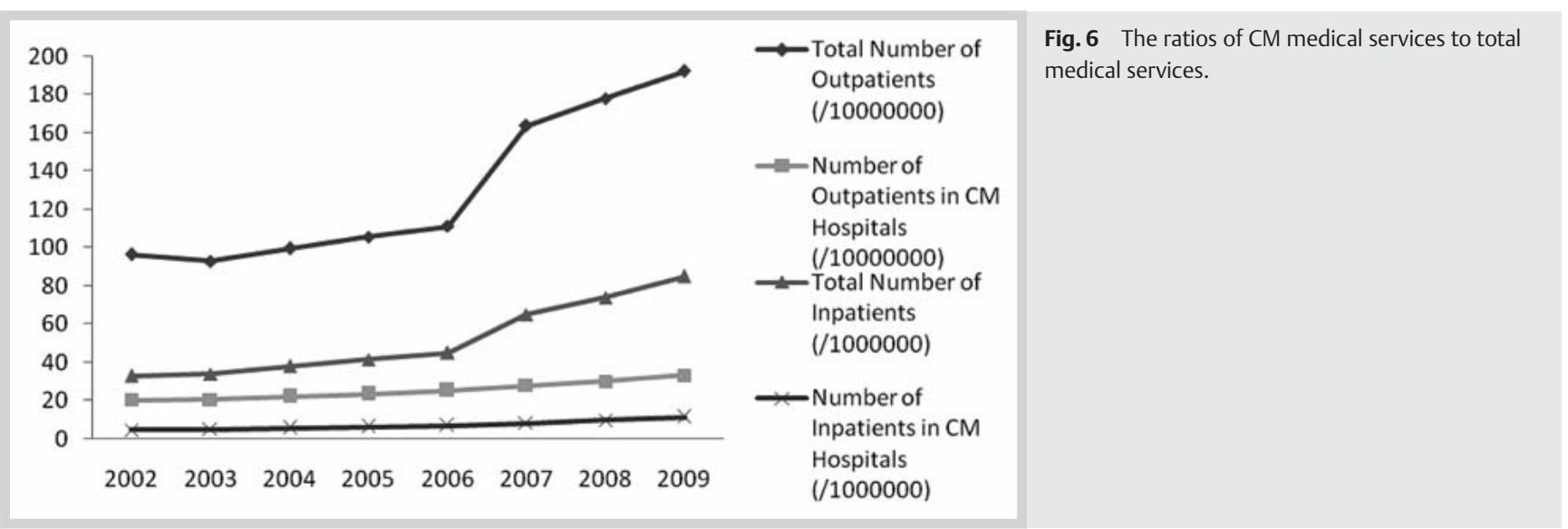

1307 items have the function of improving immunity, 780 of regulating blood lipids, 327 of regulating blood glucose, 94 are used as antioxidants, 140 for improving memory, 292 for improving sleep, 566 as antifatigue agents, 191 as anti-hypoxia agents, 222 for weight-reduction, 225 for improving osteoporosis, and 328 as purgation agents. As to the application of CMM, 856 items of health functional foods used Ginseng Radix, Ginseng rubra Radix, Codonopsis Radix, or Panacis Quinquefolii Radix as ingredients, with the main effects as immune regulators, antifatigue, and antiaging ingredients. These health products, with total sales about 40 billion RMB (Yuan), have become essential products used by patients with chronic diseases for prevention and rehabilitation.

$\mathrm{CM}$ as one part of the Chinese health care system has been favored by both patients and medical professionals. Especially patients with chronic diseases welcome CM treatment, and more medical professionals believe that CM is good at the prevention and treatment of chronic diseases. Based on the preference for $\mathrm{CM}$ in the treatment of chronic diseases, Chinese authorities encourage further application and overall development of CM in the health care system.

\section{Has Shown Efficacy in the Treatment of Chronic Diseases}

The very large clinical services of $\mathrm{CM}$ in China attract an increasing number of scientists focusing on demonstrating or providing evidence of the efficacy of $\mathrm{CM}$ for treating chronic diseases in the past 30 years. Although the interest in and use of CM is not the most valuable indication that $\mathrm{CM}$ is effective, it does show at least that patients with chronic diseases and the physicians who treat them are looking for CM therapeutic options.

More CM clinical studies have been reported (in Chinese) in China over the past 30 years ( $\odot$ Fig. 7 ), and the majority are observational ones since it is still difficult to conduct randomized controlled trials (RCTs) for CM with its unique diagnostic approach. The observational clinical studies on CM do show its efficacy for the treatment of chronic diseases. The treatment processes, which include individually prescribed, bulk-dispensed, waterbased decoctions, are the professional standard of CM care in China [8]. This means that decoctions have their own particular indications and uses in a large outpatient population. Usually, nonrandomized controlled clinical trials and observational studies indicate decoctions' efficacy while their effectiveness tested by RCTs has not yet been verified.

Recently, numerous RCTs have been conducted in China to evaluate the efficacy of PCMs generated from well-known Chinese medicine formulae or from currently effective practice formulae for the treatment of chronic diseases. Some studies have shown good effectiveness in the treatment of certain chronic diseases, such as hypertension [9], gastroenteritis [10], diabetes mellitus [11], rheumatoid arthritis [12], cerebrovascular disease [13], intervertebral disc disorders [14], chronic obstructive pulmonary disease (COPD) [15], ischemic heart disease [16], cholelith and cholecystitis [17], and peptic ulcer [18]. However, the efficacy evaluation focus on PCM is not comprehensive enough since the clinical trials are not so good to evaluate the individually pre- 


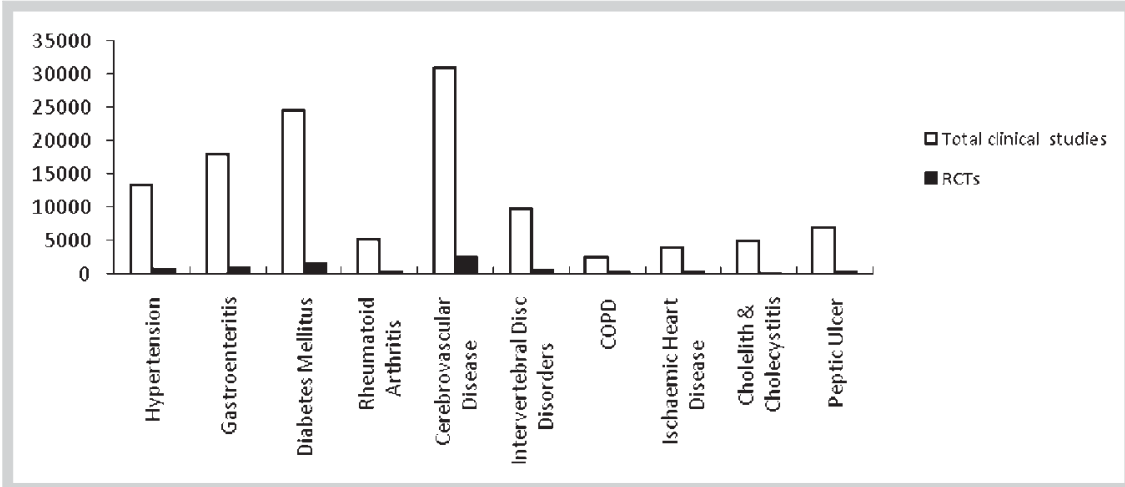

Fig. 7 The number of papers in total CM clinical studies and RCTs for 10 chronic diseases published in China from 1979 to 2009 in the Chinese BioMedical Literature Database (CBM) (http://sinomed.imicams.ac.cn/index.jsp).

scribed decoctions. In fact, the bulk of current RCTs are testing one substance's action on a specific chronic disease diagnosed with WM (biomedicine) and aimed to prove the "one size fits all" hypothesis, while CM practice is more personalized and the herbal combination is prescribed individually on the basis of each person's particular CM pattern (Zheng or syndrome).

As the evidence gathering tools, systematic reviews (SRs) and meta-analyses provide synthesis of available research. The number of SRs and meta-analysis on CM is increasing rapidly after evidence-based medicine (EBM) was introduced and practiced in China. Here, recently published findings of SRs that explore a range of evidence on $\mathrm{CM}$ for the treatment of chronic diseases have been summarized.

For herbal medicines used against irritable bowel syndrome, 75 randomized trials, involving 7957 participants are included [19]. Traditional Chinese formulae showed significant improvement of the global symptoms. 22 CMM demonstrated a statistically significant benefit in symptom improvement. Six tested PCM showed an additional benefit from the combination therapy compared to conventional monotherapy. Some herbal medicines deserve further examination in high-quality trials.

For Chinese herbal medicines used in the treatment of type 2 diabetes mellitus, 66 randomized trials, involving 8302 participants are included [20]. Methodological quality was generally low. Some PCM showed hypoglycemic effects in type 2 diabetes. Some PCM deserve further examination in high-quality trials.

Danshen (Radix Salviae Miltiorrhizae) and related PCM were introduced into clinical practice for the treatment of ischemic stroke in 1970 in China. Six trials involving 494 patients were included [21]. These Dan Shen products were associated with a significant increase in the number of patients with a positive outcome. No deaths were reported within the first two weeks of treatment or during the whole follow-up period. The authors suggest that further high-quality randomized controlled trials should be performed.

Most of the clinical studies have been shown to be inconclusive about CM for the treatment of chronic diseases [22-25]. Reasons were multiple. Most of these projects employed "standard" randomized controlled designs that were not suitable for CM trial designs, and some of the research had not involved collaboration between WM and CM researchers. Thus, there was a failure to consider only the fundamental concepts of CM. It is important to incorporate the concepts of both WM and CM into research protocols, thus collaboration between WM and CM practitioners is essential. Despite the fact that RCT is considered to be the methodology offering the highest level of evidence, different types of research are needed to answer different types of clinical ques- tions. Observational studies and case reports often are the best research methods suitable for certain clinical researches. CM efficacy research is one of them. A case report indicated effective treatment for diabetic foot ulcers with integration of $\mathrm{CM}$ and WM [26].

$\mathrm{CM}$ is holistic and conceptual, and it identifies and treats patterns rather than diseases. Because of the unique characteristics of $\mathrm{CM}$ pattern classification, CM efficacy evaluation on one herbal product or preparation should focus on a specific subgroup of patients with a specific disease. Unfortunately, many SRs on CM efficacy, which declared that there were no solid evidences to support the CM intervention efficacy and indicated that the quality of clinical trials of CM are needed for collecting evidence to support $\mathrm{CM}$ clinical application, have neglected the application of CM pattern classification [27]. For example, based on our search on China Biology Medicine (Chinese) and Pubmed (English) databases, there were 180 RCTs on Danshen herbal products, which is commonly used in clinical practice in China for activation of blood stasis (based on CM theory), and only two studies described the trial design based on CM pattern classification. Since CM pattern classification is aimed to further classify the patients into subgroups, the efficacy would be improved if the responsive cases could be distinguished from nonresponsive cases with CM patterns. Therefore, the incorporation of $\mathrm{CM}$ pattern classification into disease diagnosis, an innovative approach by additional specific diagnosis based on CM theory for documented indications, would greatly improve the assessment of the efficacy of CM interventions.

It has been reported that $\mathrm{CM}$ pattern differentiation can help to specify the indications for combination of biomedical therapy in the treatment of rheumatoid arthritis (RA), and our previous work indicated that the total effective rate of $\mathrm{CM}$ intervention could be predicted to be $80 \%$ if $10 \%$ of the patients were classified with a corresponding CM pattern [28]. This multicenter, randomized, controlled trial suggested the $\mathrm{CM}$ pattern (or CM related symptom assemblages) to have predictive roles considering the American College of Rheumatology 20\% response criteria (ACR20) evaluation in RA. Our other RCT was performed to compare the symptomatic effects on patients with benign prostatic hyperplasia (BPH) treated by two therapeutic approaches, WM and $\mathrm{CM}$, and the results showed that $\mathrm{CM}$ is a potentially effective treatment in improving the quality of life (QOL), prostate volumes, and maximum urine flow ratio (UFR) for a subgroup of patients with $\mathrm{BPH}$, and the non-urethra-related symptoms experienced by BPH patients might be one of the parameters for further distinguishing the effects of CM and WM [29]. The results suggest that it may also be useful in defining specific indications both for 
Table 1 Summary of adverse events for chronic diseases reported in SRs (CM vs. pharmaceuticals).

\begin{tabular}{|c|c|c|c|c|}
\hline Disease & Intervention & Year published & Journal & Authors' conclusions \\
\hline \multirow[t]{2}{*}{ Hypertension } & Pharmaceuticals & 2008 & $\begin{array}{l}\text { Chinese Journal of Evidence- } \\
\text { based Medicine }\end{array}$ & The incidence of adverse reaction is $9.66 \%$ [31]. \\
\hline & $\mathrm{CM}$ & 2008 & $\begin{array}{l}\text { Liaoning Journal of Traditional } \\
\text { Chinese Medicine }\end{array}$ & $\begin{array}{l}\text { The adverse effect is diarrhea, incidence rate is } \\
\text { about } 2.56 \% \text { [22]. }\end{array}$ \\
\hline \multirow[t]{2}{*}{$\begin{array}{l}\text { Intervertebral disc } \\
\text { disorders }\end{array}$} & Pharmaceuticals & 2007 & None & $\begin{array}{l}\text { Many adverse events were reported, more details in } \\
\text { the article [32]. }\end{array}$ \\
\hline & $\mathrm{CM}$ & 2009 & $\begin{array}{l}\text { Chinese Journal of Evidence- } \\
\text { based Medicine }\end{array}$ & $\begin{array}{l}\text { No adverse events reported in included studies } \\
\text { [23]. }\end{array}$ \\
\hline \multirow[t]{2}{*}{ COPD } & Pharmaceuticals & 2005 & The Cochrane Library & $\begin{array}{l}\text { There was an increased risk of adverse effects, in- } \\
\text { cluding increased blood glucose, adrenal suppres- } \\
\text { sion, and reduced serum osteocalcin [33]. }\end{array}$ \\
\hline & $\mathrm{CM}$ & 2009 & $\begin{array}{l}\text { Chinese Journal of Evidence- } \\
\text { based Medicine }\end{array}$ & $\begin{array}{l}\text { Gastrointestinal adverse events reported in } 3 \text { RCTs. } \\
\text { No serious adverse events from the herbal medi- } \\
\text { cines were reported [24]. }\end{array}$ \\
\hline \multirow[t]{2}{*}{$\begin{array}{l}\text { Ischaemic heart } \\
\text { disease }\end{array}$} & Pharmaceuticals & 2010 & Lancet & $\begin{array}{l}\text { Serious drug-related adverse events were not sig- } \\
\text { nificantly increased by fibrates ( } 17413 \text { participants, } \\
225 \text { events), although increases in serum creatinine } \\
\text { concentrations were common }(1.99,1.46-2.70 \text {; } \\
p<0.0001) \text { [34]. }\end{array}$ \\
\hline & $\mathrm{CM}$ & 2008 & $\begin{array}{l}\text { Journal of the Fourth Military } \\
\text { Medical University }\end{array}$ & $\begin{array}{l}\text { No adverse events reported in included studies } \\
\text { [23]. }\end{array}$ \\
\hline
\end{tabular}

CM interventions and biomedical therapies used for the treatment of other chronic diseases.

$\mathrm{RCT}$, as a good approach to evaluate the effectiveness of intervention, should be used widely in CM clinical study, and CM pattern classification should be incorporated into the RCT design in order to make sure that we can assess the real efficacy of $\mathrm{CM}$ in the treatment of chronic diseases. Following the active and important role of $\mathrm{CM}$ in the treatment of chronic diseases, the Chinese government continued to raise research funding for evidencebased CM, and more than fifty CM RCTs programs were supported in the "Eleventh Five-Year" plan (2006-2010) by the Ministry of Science and Technology. We believe that more positive results from RCTs with CM pattern classification will be shown up in the near future.

\section{Has Been Proved to Be Relatively Safe in the Treatment of Chronic Diseases $\nabla$}

$\mathrm{CM}$ is regarded as safe since $\mathrm{CM}$ practice has a history of several thousands of years, and it achieved personalized therapy by means of a successful organization of concerted actions derived from holistic, multitarget, and multidimensional pharmacological actions [30]. However, the safety issues of CM have been established through experience of clinical practice during the evolution and development of the traditional practice.

We searched literatures on safety and adverse drug reactions (ADRs) of CM treatment from 1978 to October of 2010 in the Sinomed database (http://sinomed.imicams.ac.cn/), and there are altogether 30631 related papers on the safety of $\mathrm{CM}$. The increasing literature number on ADRs after the year 2005 indicated the close attention paid to the safety issue of CM recently in China. - Table 1 shows the summary of adverse events for chronic diseases reported in SRs (CM vs. Pharmaceuticals) focusing on the common chronic diseases in China. The conclusion exhibited a desirable safety profile of CM compared to pharmaceuticals.

$\mathrm{CM}$ products (including CMM) could be applied according to the principles of diagnosis and treatment in CM, and based on that,
CM practice can be considered as safe. There are various studies reporting the good safety of $\mathrm{CM}$ decoctions in the treatment of some chronic diseases [35-39]. Our survey also showed that both $\mathrm{CM}$ practitioners and patients believe that decoction is effective and safe (unpublished data). Although the majority of herbal products (PCMs) have been reported to be safe [40-47], the safety issues were still more prevalent in many PCMs when compared to $\mathrm{CM}$ decoctions due to the usually long-period usage of PCM. The latest published National Adverse Drug Reaction (ADR) Report by State Food and Drug Administration (SFDA) asserted that in the year of 2009, only $13.3 \%$ ADRs were related to Chinese medicines, and totally about 2600 categories were involved in these reactions, among which, 99.5\% were relevant to PCM, less than $0.5 \%$ to decoctions. In particular, $52 \%$ of the total ADRs were related to CM injections [48]. In the past years, the safety issue on $\mathrm{CM}$ injections was a hot topic, and questions such as whether $\mathrm{CM}$ injection could be considered to be a traditional dosage form and how to set up the quality control were discussed both in scientific research and the administration field. The issues about CM injection products have aroused the high attention of the authorities [49]. The SFDA has set up a mandatory documentation system for CM injections, with such determinations as the basic ingredients in $\mathrm{CM}$ injections must be clear, the single components of the active ingredient injection should not be less than $90 \%$, and the systemic clinical trials should be a prerequisite for safety assessment [50].

To evaluate the safety of $\mathrm{CM}$ is complicated by using conventional methodological approaches since CM products are complex, which includes chemical complexity, in particular in prescriptions containing multiple herbal medicines, the lack of known synergetic active ingredients, the risk of contaminants such as pesticides, heavy metals, and the addition of other ingredients (sometimes pharmaceuticals), deterioration and variation in composition [30]. Furthermore, the interactions between CM and pharmaceutical drugs might be another issue related to the safety of CM, and it should also be addressed [51].

Any kind of medicines when used inappropriately may turn beneficial pharmacological actions into toxicity [52]. Inappropriate 
application of $\mathrm{CM}$ could lead to more ADRs in clinical practice. $\mathrm{CM}$ herbs (CMM) and $\mathrm{CM}$ products (such as herbal extract and PCM) recorded in the Chinese pharmacopoeia (version 2010) either have been used for thousands of years for prevention and treatment of diseases in China or passed a strict safety evaluation, and when applied or prescribed according to CM theory, they have been proven to be safe. Also the potent and toxic CMMs are included in the Pharmacopoeia with warning and precaution to use. In addition, all the CM products were proven to have high quality, efficacy, and safety with strict examinations, including the tests based on Good Agricultural Practice (GAP), Good Sourcing Practice (GSP), Good Laboratory Practice (GLP), Good Manufacturing Practice (GMP), and Good Clinical Practice (GCP) [53]. Unprofessional CM practice or improper application of $\mathrm{CM}$ would lead to unfavorable adverse events. For example, in clinical research, it has been reported that the side effects of a Tripterygium multiglycoside preparation in RA patients with CM dampnessheat pattern were lower than in patients with CM yin-deficiency of liver and kidney pattern [54]. One retrospective study also pinpointed that the main factors related to safety problems of the Niuhuang Jiedu tablet are irrational drug use and drug quality [55]. Thus, to ensure clinical rational application of CM and improve the quality of CM products are the major steps for safer $\mathrm{CM}$ application in clinics. On the other hand, some ADRs in CM treatment might be due to the misunderstanding of CM. In view of $\mathrm{CM}$, some ADRs can be regarded as a sign of $\mathrm{CM}$ pattern change, and CM pattern can be a predictive factor for effectiveness. Thus, it is important to explore the correlation between ADRs and efficacy. In a RCT, it was reported that gastrointestinal ADRs were inversely correlated with efficacy in conventional biomedical therapy for the treatment of RA [56]. To better achieve the goal of further analysis and comprehension on ADR, detailed and particular ADR related efficacy analysis is essential in clinical research.

CM safety profile is essential for CM application in clinical practice, especially in patients with chronic diseases since they often need prolonged treatment with CM products. Though clinical application based on CM theory and strict quality control can improve the safer application of CM, CM safety has not been fully determined, and further studies are needed to explore the mechanisms of $\mathrm{CM}$ products in order to decipher their toxic activities.

\section{Major Issues for Worldwide Application of CM}

$\nabla$

CM has been influencing the health care system in China for thousands of years, and supplies about one-fifth of the medical services nowadays in China. More importantly, CM takes an active role in preventive and rehabilitative medicine in China. Though CM has been spread worldwide recently, there are still some obstacles for further application of CM in medical services worldwide. The major issues include the $\mathrm{CM}$ specific diagnostic approaches and interventions.

\section{CM specific diagnostic approach: pattern classification}

CM pattern classification is applied to stratify the patients based on CM information. CM pattern classification has been proven to be more essential, as many CM physicians prefer to prescribe herbal formulae for patients based on this classification. Increasing numbers of medical researchers recognized that the combination of disease diagnosis in biomedicine and pattern classification in CM is essential for the clinical practice, and it has been a common practice model in China since it may produce a better clinical effect [57]. However, there are still some problems in the application of CM pattern classification for the treatment of chronic diseases in the biomedicine field.

The first is that the practice of pattern classification is very difficult, since pattern classification is mainly based on symptoms (including self-reported signs), tongue and pulse diagnosis [58]. Poor understanding in $\mathrm{CM}$ pattern classification will lead to the unsuitable application of CM products, and most of the products (such as PCMs) are used by biomedicine doctors and can be obtained from markets. Furthermore, the unsuitable application of the PCM will result in lower efficacy and even higher risks in safety. Therefore simplifying the pattern classification would be the most important issue for better understanding of $\mathrm{CM}$ by biomedicine professionals for further $\mathrm{CM}$ application. A recent study shows that the symptoms, though diversified in a disease, can be clustered into specific groups with biostatistical approaches [59]. Thus, it is possible for us to find some approaches to simplify the $\mathrm{CM}$ pattern classification and to guide the application of herbal products in future clinical practice.

The second problem is the lack of CM pattern criteria for clinical practice in the treatment of diseases. In some RCTs, patients with the same disease were recruited and received CM or conventional therapies, respectively, and the CM pattern classification was ignored. In these studies, conventional treatment tends to produce a better curative effect than CM. This should be the major reason why the RCTs failed to evaluate the real efficacy of $\mathrm{CM}$ since CM could be used only to treat part of the patients with corresponding $\mathrm{CM}$ pattern. Therefore, $\mathrm{CM}$ pattern classification criteria become the key issue both for RCT and clinical practice. Recently pattern criteria were recommended based on expert consensus and analysis of CM information obtained from the disease [60]. We believe that the CM pattern criteria, even primary criteria, could be very helpful for further application of CM products since they could be revised during the application.

$\mathrm{CM}$ pattern classification, as the key in CM diagnosis, should be well defined with biomedical language and thus CM would take a more important role in the treatment of chronic diseases worldwide.

\section{CM specific intervention: herbal mixture}

$\mathrm{CM}$ intervention using herbal formulae which consist of several herbs is highly individualized. In the past decades, more scientists have been trying to elucidate the chemistry and mechanism of CM intervention in the treatment of disease. Though many progresses have been made, there is still a long way to go in the clarification of the mechanism through which an herbal mixture works in the treatment of a disease. Furthermore, the clinical practice in $\mathrm{CM}$ abides by "multiple formulae for one disease based on the changes of CM pattern and adjusting the formulae based on the change of symptoms", and this makes it more difficult to elucidate the action mechanism of an herbal mixture.

Theoretically, there must be some core herbal combinations in the treatment of one specific disease. We propose a hierarchical analysis algorithm known as discrete derivatives, which is based on the frequency analysis of herbal combinations used in all published clinical studies in China [61]. This algorithm can retrieve simple and meaningful herbal combination networks from large data sets. Taking "RA" as an example, the "core" herbal combinations used in the treatment of RA consist of 19 CMM: Rumulus Ginnamomi (Gui Zhi), Rhizoma Anemarrhenae (Zhi Mu), Radix Angelicae Sinensis (Dang Gui), Radix Astragali Mongolici (Huang 
Qi), Radix et Rhizoma Clematidis (Wei Ling Xian), Rhizoma Chanxiong (Chuan Xiong), Radix Paeoniae Alba (Bai Shao), Radix et Rhizoma Tripterygii (Lei Gong Teng), Radix Angelicae Biserratae (Du Huo), Rhizoma et Radix Notopterygii (Qiang Huo), Olibanum (Ru Xiang), Myrrha (Mo Yao), Pheretima Aspergillum (Di Long), Radix Aconiti (Chuan Wu), Radix Glycyrrhizae (Gan Cao), Radix Stephaniae Tetrandrae (Fang Ji), Radix Aconiti Lateralis (Fu Zi), Radix Saposhnikoviae (Fang Feng), Herba Asari Mandshurici (Xi Xin). Some other CMM commonly used together with the core complex combinations are often added due to the changes of CM pattern or symptoms after initial treatment [62]. The understanding of the chemical profiles and bioactivities of the core herbal combination will help to elucidate the action mechanism of the herbal formula.

Systems biology investigations and symptoms analysis of CMM formulae can be applied as a holistic approach in analyzing their complexity. This approach has become an important direction for the elucidation of the action mechanism of herbal mixtures [63]. Our previous study showed that therapeutic efficacies of Salvia miltiorrhiza (SM) and Panax notoginseng (PN), in combination, indicate interactions between SM and PN in multiple pathways of biological processes. SM plays a principal role and PN serves as adjuvant to assist the effects during the treatment of coronary heart disease [64]. Thus, we recommend applying systems biology, including data-mining from prescription analysis and bioinformatics, for elucidating the mechanisms through which herbal mixtures work in the treatment of diseases, and studies of quality and adverse effects when CMMs are not used properly [65].

With the quality, safety, and bioactivity of CM formulae and products assured and their mechanisms elucidated, the clinical trials of these medications with patients' reported outcomes should be placed in a priority position for providing CM treatment worldwide.

\section{Conclusion}

$\nabla$

In summary, CM has been widely used in China with its convenient accessibility, affordability, efficacy, and safety in the treatment of chronic diseases. CM practice with quality practitioners and CMM products should be shared worldwide in the treatment of chronic diseases for which WM does not have adequate answers. Although CM pattern classification of diagnosis and treatment, as well as complexity of CM prescriptions may require further translation into conventional health care terms, the application of modern biomedical science, bioinformatics, and chemical profiling of CM prescriptions can be applied to provide new directions for clinical studies with evidence-based approaches for assessing CM products and practice. Eventually CM will play an important role in integrative health care worldwide.

\section{Acknowledgements}

$\nabla$

The authors acknowledge the reading of drafts of this manuscript and the technical assistance offered by Dr. Zheng Guang, Guo Hongtao, and Zha Qinglin. This study was supported jointly by the National Eleventh Five-Year Support Project of China (2006BAI04A10), National Science Foundation of China (No. 90709007, 30825047, and 30902003), and MOST Innovation Project (No. 2008IM020900).

\section{References}

1 Health China: 2020. Ministry of Public Health of China: Internal Document; 2010

2 Tang JL, Liu BY, Ma KW. Traditional Chinese medicine. Lancet 2008; 372: $1938-1940$

3 Browne $D$. The long march to primary health care in China: from collectivism to market economics. Public Health 2001; 115: 2-3

$4 \mathrm{Wu}$ C. Establishment of the community health service structure. Chin Gen Pract 2006; 9: 365-366 [in Chinese]

5 Wang J, Kushner K, Frey 3rd JJ, Ping DX, Qian N. Primary care reform in the People's Republic of China: implications for training family physicians for the world's largest country. Fam Med 2007; 39: 639-643

6 State Administration of Traditional Chinese Medicine of the People's Republic of China, School of Management in Beijing University of Chinese Medicine. China statistical yearbook of Chinese medicine. Available at http://www.satcm.gov.cn/. Accessed March 31, 2011

7 Ministry of Health of The People's Republic of China. Chinese health statistical digest 2010. Available at http://www.moh.gov.cn/publicfiles/ business/htmlfiles/zwgkzt/ptjty/digest2010/index.html?page=1/ sheet017.htm. Accessed March 31, 2011

8 Flaws $B$. The treatment of modern Western diseases with Chinese medicine. Boulder: Blue Poppy Press; 2005

9 Liu D, Lin H, Wang X, Zhou W, Chen G, Guo W, Ye J, Xu Z, Chen H, Chen XM, Lai $Z$, Xiang $C$. Influence of removing blood stasis and tonifying liver and kidney method on quality of life in patients with essential hypertension: randomized controlled observation. Chin J Clin Rehabil 2006; 10: 9-12 [in Chinese]

10 Yu Z, Wang G, Chen GY, Chang J, Zhang Y, Zhang RM. Muo-luo-dan. Concentrated pill in treatment of chronic atrophic gastritis (the stomachyin of deficiency and stagnated blood of stomach meridian): a prospective, randomized, controlled trial. West China Med J 2007; 22: $287-$ 289 [in Chinese]

11 Tong XL, Ni Q Lian FM, Hu JQ Yu FH, Ou Y, Xue Q Jiang Z, Wu ST, Jiang H, Gao TS, Yao C, Zhao M, Zhang Y. Multi-center randomized controlled and double blind trial of Tang-min-ling pills in the treatment of type 2 diabetes mellitus. Chin J Clin Pharmacol 2009; 25: 104-108 [in Chinese]

12 Guo FL, Han C, Liu QP, Liu LJ. Clinical observation on the effect of Tongbi Heji plus syndrome differentiation treatment for 90 rheumatoid arthritis patients. J Tradit Chin Med 2008; 49: 897-900 [in Chinese]

13 Zhou CX, Cui X, Li XQ Zeng HL, Ni HH, Huang CS, Wu J, Shi JC, Feng ML, $W u$ YG. Clinical research on combined herbal medicine and acupuncture in treating poststroke depression. Shanghai J Tradit Chin Med 2010; 44: 46-48 [in Chinese]

14 Qin $X Y$, Li XX, Suteanu S. Comparative study on Chinese medicine and Western medicine for treatment of prolapse of lumbar intervertebral disc. Chin Acupunct Moxibustion 2007; 27: 365-368 [in Chinese]

$15 \mathrm{Xu} Y H$, Wang JH, Yang FX, He XZ. The effects of respiratory rehabilitation training on pulmonary function and quality of life of patients with COPD. Chin J Rehabil 2010; 25: 120-122 [in Chinese]

16 Qing H, Wang SF, Fan JM, Zha LH, Mao JY, Sun LJ, Zhang RL. Multi-center clinical study of Rhadiola extract injection on the treatment of stable angina pectoris of coronary heart disease with cariac blood stasis syndrome. Chin Tradit Pat Med 2009; 31: 343-346 [in Chinese]

17 Liu T, Liu C, Gao PX, Yu CP. Clinical study on method of "tonifying the lung to check the liver" for treatment of chronic cholecystitis. Chin Acupunct Moxibustion 2005; 25: 855-856 [in Chinese]

18 Wei JQ Lin SR, He WN. Compared with tagamet in treating chronic gastric ulcer. Chin J Integr Tradit West Med 1994; 14: 17-18 [in Chinese]

19 Liu JP, Yang M, Liu YX, Wei ML, Grimsgaard S. Herbal medicines for treatment of irritable bowel syndrome. Cochrane Database Syst Rev 2006; 1: CD004116

20 Liu JP, Zhang M, Wang WY, Grimsgaard S. Chinese herbal medicines for type 2 diabetes mellitus. Cochrane Database Syst Rev 2004; 3: CD003642

$21 \mathrm{Wu}$ B, Liu M, Zhang S. Dan Shen agents for acute ischaemic stroke. Cochrane Database Syst Rev 2007; 2: CD004295

22 Wang H, Shang HC, Zhang JH, Chen J, Sun BZ, Shang DD, Xiang YZ, Cao HB, Ren M, Cuo LP, Zhang BL. Niuhuang Jiangya preparation for treatment of essential hypertension: a systematic review. Liaoning J Trad Chin Med 2008; 35: 649-652 [in Chinese]

23 Wang SZ, Lin HL, Song HM, Zhong WH, Wu TX, Liu GJ, Chen SQ. Conservative in the treatment of protrusion of cervical vertebra intervertebral disc: a systematic review. Chin J Evid Based Med. 2009; 9: 331-336 [in Chinese] 
24 Zhou W, Zhong YQ Yang HM, Jiang HL, Fu JJ, Zhang WB, Mao B. Traditional Chinese medicine in the treatment of chronic obstructive pulmonary disease in stable stage: a systematic review of randomized controlled trials. Chin J Evid Based Med 2009; 9: 311-318 [in Chinese]

25 Jin X, Wang ZR. Meta analysis of clinical research literature on supplementing qi and activating blood circulation for coronary heart disease. J Fourth Military Med Univ 2008; 29: 1188-1190 [in Chinese]

26 Manheimer E, Wieland S, Kimbrough E, Cheng K, Berman BM. Evidence from the Cochrane Collaboration for Traditional Chinese Medicine therapies. J Alternat Complement Med 2009; 15: 1001-1014

27 Xie XS, Wang YJ, Zuo C, Fan JM, Li XJ. A case report of an effective treatment for diabetic foot ulcers with integration of traditional Chinese medicine and Western medicine. J Diabetes Complications 2009; 23: 360-364

28 Zha QL, He YT, Ding XR, Jiang M, Liu XW, Lu C, Tsang I, Lu AP. The predictive role of symptoms/signs on ACR20 responses in rheumatoid arthritis analyzed with data mining approaches. Proceedings of the 2nd International Conference on BioMedical Engineering and Informatics, BMEI, Tianjin, China; 2009: 1-8

29 Li S, Lu A, Wang Y. Symptomatic comparison in efficacy on patients with benign prostatic hyperplasia treated with two therapeutic approaches. Complement Ther Med 2010; 18: 21-27

30 Chan $K$. Chinese medicinal materials and their interface with Western medical concepts. J Ethnopharmacol 2005; 96: 1-18

31 Yi HG, Huang GZ, Liu GJ. Efficacy and safety of levoamlodipine besylate for essential hypertension: a systematic review. Chin J Evid Based Med 2008; 8: 543-550 [in Chinese]

32 Vipond $N$. Intervertebral disc replacement. Available at http://www. acc.co.nz/PRD_EXT_CSMP/groups/external_communications/documents/reports_results/prd_ctrb091239.pdf. Accessed Sep 28, 2010

33 Walters JA, Walters EH, Wood-Baker R. Oral corticosteroids for stable chronic obstructive pulmonary disease. Cochrane Database Syst Rev 2005; 3: CD005374

34 Jun M, Foote C, Lv J, Neal B, Patel A, Nicholls SJ, Grobbee DE, Cass A, Chalmers J, Perkovic V. Effects of fibrates on cardiovascular outcomes: a systematic review and meta-analysis. Lancet 2010; 375: 1875-1884

35 Sze SC, Wong KL, Liu WK, Ng TB, Wong JH, Cheung HP, Yow CM, Chu ES, Liu $\mathrm{Q}$ Hu YM, Tsang KW, Lee WS, Tong Y. Regulation of $\mathrm{p} 21$, MMP-1, and MDR-1 expression in human colon carcinoma HT29 by Tian Xian liquid, a Chinese medicine formula, in vitro and in vivo. Integr Cancer Ther, advance online publication August 11, 2010; PMID: 20702488

36 Zhou CY, Tang JY, Fang DY. Clinical study on active rheumatoid arthritis treated with simiao xiaobi decoction. Zhongguo Zhong Xi Yi Jie He Za Zhi 2010; 30: 275-279

37 Hsieh SC, Lai JN, Chen PC, Chen CC, Chen HJ, Wang JD. Is Duhuo Jisheng Tang containing Xixin safe? A four-week safety study. Chin Med 2010; 5: 6

38 Geng $Q$ Lu BD, Huang XJ. Effect of modified tianxiong powder in treating oligospermia and asthenospermia. Zhongguo Zhong Xi Yi Jie He Za Zhi 2010; 30: 496-498

39 Zhu J, Wan X, Zhu Y, Ma X, Zheng Y, Zhang T. Evaluation of salidroside in vitro and in vivo genotoxicity. Drug Chem Toxicol 2010; 33: 220-226

40 Zhao L, Li AY, Lv H, Liu FY, Qi FH. Traditional Chinese medicine Ningdong granule: the beneficial effects in Tourette's disorder. J Int Med Res 2010; 38: 169-175

41 Xie JX, Hu HT, Xiao DN, Xie HZ, Zhu CB, Yang WT, Huang XX, He XJ, Liang GR, Wang ZG. Safety and efficacy of Qianliean suppository for chronic prostatitis of damp-heat and blood-stasis syndrome: a randomized, single-blind, parallel controlled, multi-centered phase III clinical trial. Zhonghua Nan Ke Xue 2009; 15: 1049-1052

42 Jia G, Meng MB, Huang ZW, Qing X, Lei W, Yang XN, Liu SS, Diao JC, Hu SY, Lin BH, Zhang RM. Treatment of functional constipation with the Yunchang capsule: a double-blind, randomized, placebo-controlled, doseescalation trial. J Gastroenterol Hepatol 2010; 25: 487-493

43 Deng $M$, Sui XQ Zhu SB, Ma W, Xu Y, Chen ZM. Clinical observation on the treatment of atrial fibrillation with amiodarone combined with Shenmai Injection. Chin J Integr Med 2010; 16: 453-456

44 Fan AY, Lao L, Zhang RX, Zhou AN, Berman BM. Preclinical safety evaluation of the aqueous acetone extract of Chinese herbal formula modified Huo Luo Xiao Ling Dan. Zhong Xi Yi Jie He Xue Bao 2010; 8: 438-447
45 Xu DY, Shu J, Huang QY, Liu L, Zhao SP. A comparative study of the efficacy and safety Zhibitai and atorvastatin. Zhonghua Nei Ke Za Zhi 2010; 49: 392-395

46 Xu DY, Shu J, Huang QY, Wasti B, Chen C, Liu L, Zhao SP. Evaluation of the lipid lowering ability, anti-inflammatory effects and clinical safety of intensive therapy with Zhibitai, a Chinese traditional medicine. Atherosclerosis 2010; 211: 237-241

47 Wang L, Zhang RM, Liu GY, Wei BL, Wang Y, Cai HY, Li FS, Xu YL, Zheng SP, Wang $G$. Chinese herbs in treatment of influenza: a randomized, double-blind, placebo-controlled trial. Respir Med 2010; 104: 1362-1369

48 State Food and Drug Administration. 2009 national annual report of adverse drug reaction surveillance. Available at http://www.sda.gov.cn/ WS01/CL0051/48201.html. Accessed March 31, 2011

49 Xiong XJ, Wang J, He QY. Application status and safety countermeasures of traditional Chinese medicine injections. Zhong Xi Yi Jie He Xue Bao 2010; 8: 307-311

50 State Food and Drug Administration. Notification on the 7 technical guidelines for the production processes and re-evaluation of the safety of Chinese medicine injection. Available at http://www.sda.gov.cn/ WS01/CL0058/54917.html. Accessed March 31, 2011

51 Chan K, Cheung $L$. Interactions between Chinese herbal medicinal products and orthodox drugs. Reading: Harwood Academic Publishers, The Gordon \& Breach Publishing Group; 2000

52 Wang J, van der Heijden R, Spruit S, Hankermeier T, Chan $K$, van der Greef $J$, Xu G, Wang M. Quality and safety of Chinese herbal medicines guided by a systems biology perspective. J Ethnopharmacol 2009; 126: 31-41

53 Chan $K$. Some aspects of toxic contaminants in herbal medicines. Chemosphere 2003; 52: 1361-1371

54 Zhou J, Zhu Q Yang X. Clinical observation of side effects of Tripterygium preparation. Zhongguo Zhong Xi Yi Jie He Za Zhi 1999; 19: 77-79

55 Tong Y, Zhang L, Yang J, Yang L, Wang Y, Qu J, Zhang C. Retrospective study of adverse reactions of Niuhuang Jiedu tablet (pill) and risk control based on literature analysis. Zhongguo Zhong Yao Za Zhi 2010; 35: $1342-1345$

56 Jiang $M$, Zhao J, Lu A, Zha $Q$ He Y. Does gastrointestinal adverse drug reaction influence therapeutic effect in the treatment of rheumatoid arthritis? J Alternat Complement Med 2010; 16: 143-144

57 Lu AP, Ding XR, Chen KJ. Current situation and progress in integrative medicine in China. Chin J Integr Med 2008; 14: 234-240

$58 \mathrm{Lu} A$, Jia HW, Xiao C, Lu QP. Theory of traditional Chinese medicine and therapeutic method of diseases. World J Gastroenterol 2004; 10: 1854-1856

59 He Y, Lu A, Zha Y, Yan X, Song Y, Zeng S, Liu W, Zhu W, Su L, Feng X, Qian X, $L u C$. Correlations between symptoms as assessed in traditional Chinese medicine (TCM) and ACR20 efficacy response: a comparison study in 396 patients with rheumatoid arthritis treated with TCM or Western medicine. J Clin Rheumatol 2007; 13: 317-321

60 Jiang M, Yang J, Zhang C, Liu B, Chan K, Cao H, Lu A. Clinical studies with traditional Chinese medicine in the past decade and future research and development. Planta Med 2010; 76: 2048-2064

61 Tan Y, Guo HT, Zheng G, Zhang C, Yang J, Lu C, Zha QL, Jiang M, Lu APl. Based on text mining techniques to explore medication regularities of disease treatment with traditional Chinese medicine. World Science and Technology/Modernization of Traditional Chinese Medicine and Materia Medica 2010; 12: 823-827 [in Chinese]

62 Lu YB, Li L, Wang Z, Zheng G, Guo HT, Jiang M, Lu APl. Medication regularity of tradtional Chinese medicine against rheumatoid arthritis, gout and osteoarthritis. World Science and Technology/Modernization of Traditional Chinese Medicine and Materia Medica 2010; 12: 833836 [in Chinese]

$63 \mathrm{Lu}$ AP, Li S. Principle and application of Chinese medicine syndrome differentiation and disease classification. Zhongguo Zhong Xi Yi Jie He Za Zhi 2010; 30: 84-86

64 Chen G, Liu B, Jiang M, Tan Y, Lu AP. Systems biology approaches predict functional networks for Salvia miltiorrhiza and Panax notoginseng in combination. J Med Plant Res, in press

65 Wang JS, Van der Heijden R, Spruit S, Hankermeier, Chan K, Van der Greef J, Xu GW, Wang M. Quality and safety of Chinese medicine herbal medicines guided by a systems biology perspective. J Ethnopharmacol $2009 \cdot 126 \cdot 31-41$ 